\title{
MACHETE: A transit Imaging Atmospheric Cherenkov Telescope to survey half of the Very High Energy $\gamma$-ray sky
}

\author{
Rubén López-Coto, Juan Cortina and Abelardo Moralejo
}

Institut de Fisica dAltes Energies (IFAE), The Barcelona Institute of Science and Technology, Campus UAB, 08193 Bellaterra (Barcelona) Spain

email: rlopez@ifae.es

\begin{abstract}
Current Cherenkov Telescopes for VHE gamma ray astrophysics are pointing instruments with a field of view up to a few tens of $\mathrm{deg}^{2}$. We propose to build an array of two non-steerable telescopes with a FoV of $5 \times 60 \mathrm{deg}^{2}$ oriented along the meridian. Roughly half of the sky drifts through this FoV in a year. We have performed a MC simulation to estimate the performance of this instrument, which we dub MACHETE. The sensitivity that MACHETE would achieve after 5 years of operation for every source in this half of the sky is comparable to the sensitivity that a current IACT achieves for a specific source after a $50 \mathrm{~h}$ devoted observation. The analysis energy threshold would be $150 \mathrm{GeV}$ and the angular resolution 0.1 deg. For astronomical objects that transit over MACHETE for a specific night, it would achieve an integral sensitivity of $12 \%$ of Crab in a night. This makes MACHETE a powerful tool to trigger observations of variable sources at VHE or any other wavelengths.
\end{abstract}

Keywords. gamma rays, Imaging Atmospheric Cherenkov telescopes

\section{Introduction}

Very High Energy gamma rays are detected using space-based or ground-based detectors. From space Fermi-LAT is performing the deepest survey to date of the $\gamma$-ray sky from $20 \mathrm{MeV}$ up to energies in excess of $100 \mathrm{GeV}$, although with limited sensitivity above $10 \mathrm{GeV}$ due to its relatively small collection area $\left(0.8 \mathrm{~m}^{2}\right)$. From the ground IACTs, such as the MAGIC, H.E.S.S. or VERITAS arrays, detect gamma rays with energies above 50 $\mathrm{GeV}$ and have collection areas of more than $105 \mathrm{~m}^{2}$. They are pointing instruments with a Field of View (FoV) on the order of tens of $\operatorname{deg}^{2}$. The $12 \mathrm{~m}$ diameter Medium-Sized Telescopes in the Cherenkov Telescope Array (CTA), currently under design, have a FoV of around $60 \mathrm{deg}^{2}$. On the other hand air-shower instruments such as Milagro, Tibet and HAWC detect gamma rays at higher energies, have a comparable collection area of 80000 $\mathrm{m}^{2}$, but with a much larger FoV of $5000 \mathrm{deg}^{2}$ and high duty cycle. They are non-tracking instruments. Unfortunately they are not as efficient as IACTs in eliminating the cosmic ray background, so they suffer from a lower sensitivity and they have poorer angular or spectral resolutions. We propose to build an array of two non-steerable IACTs with a wide FoV of $300 \mathrm{deg}^{2}$. We call this array Meridian Atmospheric CHErenkov TElescope (MACHETE). The reader may find a detailed description of the instrument, its performance and its physics goals in Cortina et al. 2016. Here we focus mainly in the optics, review the performance that is estimated using a full Monte Carlo simulation and go through some of its possible applications. 


\section{Wide FoV telescopes}

A Schmidt telescope is a well-known solution to achieve a large FoV with a small focal ratio. The optical components are an easy-to-manufacture spherical primary mirror, and an aspherical correcting lens, known as a Schmidt corrector plate, located at the center of curvature of the primary mirror. The corrector plate reduces optical aberrations and at the same time acts as a stop which defines the aperture of the telescope. Our concept is inspired by a Schmidt telescope, but we have aimed at simplifying it so that it is easy and cheap to implement:

- Like in the original Schmidt telescope, the shape of the primary mirror is spherical. The nominal focal length is half of the radius of curvature.

- Also like a Schmidt telescope, the shape of the focal plane is spherical and concentric with the mirror.

- An IACT is not as stringent as an optical telescope in terms of mirror Point Spread Function (PSF). A PSF on the order of $0.05 \mathrm{deg}$ is good enough. We shall remove the corrector plate and achieve an acceptable PSF by increasing the focal ratio.

- However if we eliminate the corrector plate we are not only worsening the optical performance of the instrument, but also eliminating the stop. We must find an alternative way to limit the aperture. Compared to optical telescopes IACTs are in fact peculiar because each pixel is typically implemented as a light concentrator followed by the actual photodetector. In a natural way light concentrators can be used to define the section of the mirror which is viewed by each pixel and effectively the aperture.

The details in the optical design of MACHETE can be found in Cortina et al. 2016 . Here we will only state that MACHETE will be composed of two instruments to improve the stereoscopic reconstruction of the $\gamma$-ray images. Each of them will have a section of spherical mirror (34 m curvature) covering a region of $5^{\circ} \times 60^{\circ}$ of sky.

\section{Expected performance}

We performed a Monte Carlo simulation of the MACHETE system to evaluate its performance. In this proceeding we only describe its results, for further details on design and performance, refer to Cortina et al. 2016. Here you can find a summary of the main results we obtained for the performance of the instrument:

- The sensitivity of MACHETE is significantly better than the sensitivity of HAWC for the same observation time and energies below $5 \mathrm{TeV}$. In addition, even one year of MACHETE has better sensitivity than 5 years of HAWC below $2 \mathrm{TeV}$. In addition, the angular and spectral resolution would be significantly better than those of HAWC.

- We have taken into account the change of acceptance as a function of position of the source in the FoV. The best integral sensitivity of MACHETE is reached at $500 \mathrm{GeV}$ and it is $0.77 \%$ of the Crab flux at $30 \mathrm{deg}$ declination after 5 years of operation.

- The sensitivity of MACHETE can also be compared to the sensitivity of the planned extragalactic scan of CTA. For the full CTA-South array and an observation of half of the sky for 1000 hours, the expected integral sensitivity of the survey is $0.6 \%$ of the Crab flux above $125 \mathrm{GeV}$. MACHETE achieves a similar sensitivity in 5 years of operation at a slightly higher energy but for the same fraction of the sky.

- MACHETE would achieve an integral sensitivity of $12 \%$ of the Crab flux in a single night for all sources in the fraction of the sky that is observable in that specific night.

\section{References}

Cortina, J., López-Coto, R., \& Moralejo, A. 2016, APh, 72, 46-54 